\title{
STOCHASTICITY OF BIAS AND NONLOCALITY OF GALAXY FORMATION: LINEAR SCALES
}

\author{
Takahiko Matsubara \\ Department of Physics and Astronomy, The Johns Hopkins University, 3400 N.Charles \\ Street, Baltimore, MD 21218; and \\ Department of Physics, The University of Tokyo, Hongo 7-3-1, Tokyo 113-0033, Japan; and \\ Research Center for the Early Universe, Faculty of Science, The University of Tokyo, \\ Tokyo 113-0033, Japan.
}

\begin{abstract}
If one wants to represent the galaxy number density at some point in terms of only the mass density at the same point, there appears the stochasticity in such a relation, which is referred to as "stochastic bias". The stochasticity is there because the galaxy number density is not merely a local function of a mass density field, but it is a nonlocal functional, instead. Thus, the phenomenological stochasticity of the bias should be accounted for by nonlocal features of galaxy formation processes. Based on mathematical arguments, we show that there are simple relations between biasing and nonlocality on linear scales of density fluctuations, and that the stochasticity in Fourier space does not exist on linear scales under a certain condition, even if the galaxy formation itself is a complex nonlinear and nonlocal precess. The stochasticity in real space, however, arise from the scale-dependence of bias parameter, $b$. As examples, we derive the stochastic bias parameters of simple nonlocal models of galaxy formation, i.e., the local Lagrangian bias models, the cooperative model, and the peak model. We show that the stochasticity in real space is also weak, except on the scales of nonlocality of the galaxy formation. Therefore, we do not have to worry too much about the stochasticity on linear scales, especially in Fourier space, even if we do not know the details of galaxy formation process.
\end{abstract}

Subject headings: cosmology: theory — galaxies: statistics — large-scale structure of universe

\section{INTRODUCTION}

Probing the statistical properties of the large-scale structure of the universe has a great importance in studying the origin of our universe. Recent galaxy surveys have been 
revealing the statistical properties of distribution of galaxies on very large scales. The number density of galaxies are not necessarily proportional to the density of mass, and this ambiguity is known as galaxy biasing problem (Kaiser 1984; Davis et al. 1985; Bardeen et al. 1986). Since galaxies of different types have different clustering properties (e.g., Dressler 1980; Davis \& Geller 1976; Giovanelli, Haynes, \& Chincarini 1986; Santiago \& Strauss 1992; Loveday et al. 1996; Hermit et al. 1996; Guzzo et al. 1997), not all types of galaxies can simultaneously be unbiased tracers of mass. This ambiguity is undesirable in extracting cosmological information from the data of galaxy distribution.

The simplest model for the galaxy bias is the local, linear bias. In this simple model, the number density field of galaxy with a fixed smoothing scale $R$ is assumed to be proportional to density field of mass with a same smoothing scale:

$$
\delta_{\mathrm{g}}(\boldsymbol{x} ; R)=b \delta_{\mathrm{m}}(\boldsymbol{x} ; R),
$$

where $\delta_{\mathrm{g}}$ and $\delta_{\mathrm{m}}$ are density contrast of galaxy and of mass, respectively, with a fixed smoothing length $R$. This model is viable if (a) $\delta_{\mathrm{g}}(\boldsymbol{x} ; R)$ is dependent only on $\delta_{\mathrm{m}}(\boldsymbol{x} ; R)$ and (b) density contrast of mass is sufficiently small. The latter condition (b) is achieved by considering large scales on which density fluctuations are small enough so that only linear term becomes significant. The linear coefficient $b$ is called as bias parameter. The bias parameter is often assumed to be a constant, although it can depend on $R$ in general. The effect of nonlinearity should be taken into account when we are interested in nonlinear scales. On weakly nonlinear scale, this affect the estimation of higher order statistics (e.g., Fry \& Gaztañaga 1993).

Although the latter condition is reasonably considered to be valid if we are interested in linear scales, the former condition (a) is not trivial so far. The non-triviality of condition (a) leads us to a concept of stochastic bias which is recently argued (Dekel \& Lahav 1998; Pen 1998; Tegmark \& Peebles 1998; Tegmark \& Blomley 1998; Taruya, Koyama \& Soda 1998; Taruya \& Soda 1998; Blanton et al. 1998). In the stochastic biasing scheme, $\delta_{\mathrm{g}}(\boldsymbol{x} ; R)$ is not supposed to be determined solely by $\delta_{\mathrm{m}}(\boldsymbol{x} ; R)$, but the scatter in $\delta_{\mathrm{g}}-\delta_{\mathrm{m}}$ relation is taken into account. In linear regime in which the density contrast of mass $\delta_{\mathrm{m}}$ is small enough, and is approximated by random Gaussian field, the two-point statistics fully characterize the statistics of the scatter. In literatures, the bias parameter $b$ and the dimensionless cross correlation $r$ are used to characterize the linear stochastic biasing scheme:

$$
b(R)=\sqrt{\frac{\left\langle\delta_{\mathrm{g}}(\boldsymbol{x} ; R)^{2}\right\rangle}{\left\langle\delta_{\mathrm{m}}(\boldsymbol{x} ; R)^{2}\right\rangle}}, \quad r(R)=\frac{\left\langle\delta_{\mathrm{m}}(\boldsymbol{x} ; R) \delta_{\mathrm{g}}(\boldsymbol{x} ; R)\right\rangle}{\sigma_{\mathrm{m}}(R) \sigma_{\mathrm{g}}(R)},
$$

where $\sigma_{\mathrm{m}}=\left\langle\delta_{\mathrm{m}}^{2}\right\rangle^{1 / 2}$ and $\sigma_{\mathrm{g}}=\left\langle\delta_{\mathrm{g}}^{2}\right\rangle^{1 / 2}=b \sigma_{\mathrm{m}}$ are rms density fluctuations of mass and galaxies, respectively. 
The bias parameter $b$ in the equation (1.2) is a generalization of the bias parameter in linear deterministic biasing scheme of equation (1.1). In deterministic case, the cross correlation $r$ is always unity. From Schwarz inequality, $r$ cannot exceed unity and $r=1$ means that biasing is deterministic, $\delta_{\mathrm{g}} \propto \delta_{\mathrm{m}}$. Thus, the deviation from $r=1$ measures the stochasticity. If the smoothing scale $R$ is large enough so that $\delta_{\mathrm{m}}$ and $\delta_{\mathrm{g}}$ are considered as bivariate Gaussian field, these three parameters $b, r$ and $\sigma_{\mathrm{m}}$ contain all the statistical information about the stochastic biasing.

In literatures, these parameters are sometimes considered as free parameters to be determined by observation. However, if we could know the process of galaxy formation in detail, the parameters $b$ and $r$ would be derived from some fundamental physical processes. This is because the bias and its stochasticity come from our ignorance of the galaxy formation. Therefore, there should be some theoretical constraint between $b$ and $r$. At first sight, it seems difficult to find any constraint as we do not exactly know the process of galaxy formation. This is true especially on small scales where the nonlinear and nonlocal characters of galaxy formation plays an important role on the statistics of galaxy distributions. This problem is one of the most important issues in astrophysics and much numerical and analytical work is needed (e.g., Rees \& Ostriker 1977; White \& Frenk 1991; Cen \& Ostriker 1992; Mo \& White 1996). However, on large scales, such undesirable characters can be expected to be small. Scherrer \& Weinberg (1998), based on the local biasing scheme, showed that the stochasticity actually vanishes on large scales and galaxy autocorrelation function behaves exactly as in deterministic biasing scheme. Dekel \& Lahav (1999) also imply the same property based on a specific simple model.

In this paper, based on a general nonlocal method, we show that stochasticity in Fourier space asymptotically vanishes on linear scales under a certain condition, explicitly deriving the relation between the stochastic parameters $b, r$ and the nonlinear, nonlocal functional form of galaxy formation. We will show the first coefficient of generalized Wiener-Hermite functional, which is defined below, of the nonlocal, nonlinear relations of galaxy formation will contribute to the galaxy statistics on large scales, if that coefficient does not vanish.

In the derivation, we can use the technique developed in Matsubara (1995), in which the diagrammatic methods for the calculation of general nonlocal biasing are introduced. The diagrammatics are useful especially when the non-Gaussianity and/or higher-order correlations are interested in. In this paper, however, we derive the result without employing diagrammatics for self-consistency and for the simplicity of the problem. The diagrammatics make it easier to generalize the present results to higher-order statistics.

In $\S 2$, we revisit the mathematical methods for nonlinear, and nonlocal bias, which have been developed by Matsubara (1995). Then we derive the relation between the stochastic 
bias parameters and the nonlocality of biasing. In $\S 3$, we examine three types of biasing schemes, i.e., the local Lagrangian bias models, the cooperative model, and the peak model, according to the result of $\S 2$. In $\S 4$, we discuss the results and present the conclusions.

\section{NONLOCAL BIAS AND STOCHASTICITY}

\subsection{Mathematical Formulation of Nonlocal Bias}

In the present paradigm, the distribution of galaxies is determined by initial density fluctuations. Whether a galaxy has formed at some place or not should be fully determined by the initial fluctuations. In this sense, the galaxy formation is deterministic, although it nonlinearly, nonlocally, and possibly chaotically depends on initial density fluctuations. In the stochastic biasing scheme, this complex features of nonlinearity and nonlocality of the galaxy formation are expressed by phenomenological scatter of local $\delta_{\mathrm{g}}-\delta_{\mathrm{m}}$ relation. Thus, in principle, stochasticity can be determined by nonlinear and nonlocal deterministic processes of galaxy formation.

Since both the present mass density field $\rho_{\mathrm{m}}$ and the density field of galaxies $\rho_{\mathrm{g}}$ are determined by initial density fluctuations $\delta_{\mathrm{i}}$, they are expressed by functionals $F_{\mathrm{m}}$ and $F_{\mathrm{g}}$. In the following, instead of the initial fluctuations $\delta_{\mathrm{i}}$, we will alternatively use linearly extrapolated density fluctuations $\delta_{\mathrm{L}}=D \delta_{\mathrm{i}}$, where $D$ is a linear growth rate. The variable $\delta_{\mathrm{L}}$ is simply a linear extrapolation of the evolution of density contrast, regardless of whether or not the small scale fluctuations are actually in linear regime. The introduction of the linearly extrapolated field is just for convenience and it simply represents the initial density fluctuations. In this notation, one can write the relations as

$$
\rho_{\mathrm{m}}(\boldsymbol{x})=F_{\mathrm{m}}\left(\left[\delta_{\mathrm{L}}\right], \boldsymbol{x}\right), \quad \rho_{\mathrm{g}}(\boldsymbol{x})=F_{\mathrm{g}}\left(\left[\delta_{\mathrm{L}}\right], \boldsymbol{x}\right),
$$

where we introduce the notation $\left[\delta_{\mathrm{L}}\right]$ which means that $F_{\mathrm{m}}$ and $F_{\mathrm{g}}$ are nonlocal (and, of course, nonlinear) functionals of $\delta_{\mathrm{L}}$. These functionals also depend on the position, $\boldsymbol{x}$. It is sometimes more convenient to use the density contrast of $\rho_{\mathrm{m}}$ and $\rho_{\mathrm{g}}$ :

$$
\delta_{\mathrm{m}}(\boldsymbol{x})=\frac{F_{\mathrm{m}}\left(\left[\delta_{\mathrm{L}}\right], \boldsymbol{x}\right)}{\left\langle F_{\mathrm{m}}\left(\left[\delta_{\mathrm{L}}\right], \boldsymbol{x}\right)\right\rangle}-1, \quad \delta_{\mathrm{g}}(\boldsymbol{x})=\frac{F_{\mathrm{g}}\left(\left[\delta_{\mathrm{L}}\right], \boldsymbol{x}\right)}{\left\langle F_{\mathrm{g}}\left(\left[\delta_{\mathrm{L}}\right], \boldsymbol{x}\right)\right\rangle}-1 .
$$

One may want to expand these functional by Taylor series of $\delta_{\mathrm{L}}$, as in usual perturbative approach. However, this approach would be useful only if the nonlinearities on all scales were small, and/or galaxy formation process were linear or quasi-linear. In reality, however, the galaxy formation process is a highly nonlinear process, and it depends on the nonlinear 
behavior of mass density field in a very complex way. Thus, we cannot use Taylor expansion in our treatment below. Alternatively, we will find below that the expansion of these functional by orthogonal functionals, i.e., generalized Wiener-Hermite functionals, is useful for our purpose. The $n$-th order generalized Wiener-Hermite functional $\mathcal{H}^{(n)}$, which is introduced in Matsubara (1995) for the first time, is defined by

$$
\begin{aligned}
\mathcal{H}^{(n)}\left(\boldsymbol{x}_{1}, \ldots, \boldsymbol{x}_{n}\right)=\exp \left[\frac{1}{2} \int d^{3} x d^{3} y \delta_{\mathrm{L}}(\boldsymbol{x}) \xi_{\mathrm{L}}^{-1}(\boldsymbol{x}, \boldsymbol{y}) \delta_{\mathrm{L}}(\boldsymbol{y})\right] \\
\times \frac{(-1)^{n} \delta^{n}}{\delta \delta_{\mathrm{L}}\left(\boldsymbol{x}_{1}\right) \cdots \delta \delta_{\mathrm{L}}\left(\boldsymbol{x}_{n}\right)} \exp \left[-\frac{1}{2} \int d^{3} x d^{3} y \delta_{\mathrm{L}}(\boldsymbol{x}) \xi_{\mathrm{L}}^{-1}(\boldsymbol{x}, \boldsymbol{y}) \delta_{\mathrm{L}}(\boldsymbol{y})\right],
\end{aligned}
$$

where $\delta / \delta \delta_{\mathrm{L}}$ is a functional derivative with regard to $\delta_{\mathrm{L}}$, and $\xi_{\mathrm{L}}$ is a two-point correlation function of linear density field, $\xi_{\mathrm{L}}(\boldsymbol{x}, \boldsymbol{y})=\left\langle\delta_{\mathrm{L}}(\boldsymbol{x}) \delta_{\mathrm{L}}(\boldsymbol{y})\right\rangle$. The inverse of correlation function, $\xi_{\mathrm{L}}^{-1}$, is defined formally by

$$
\int d^{3} y \xi_{\mathrm{L}}^{-1}(\boldsymbol{x}, \boldsymbol{y}) \xi_{\mathrm{L}}(\boldsymbol{y}, \boldsymbol{z})=\delta^{3}(\boldsymbol{x}-\boldsymbol{z})
$$

The original Wiener-Hermite functional is the special case when the correlation function is identical to Dirac delta function, $\xi_{\mathrm{L}}(\boldsymbol{x}, \boldsymbol{y})=\delta^{3}(\boldsymbol{x}-\boldsymbol{y})$. If $\delta_{\mathrm{L}}$ has only finite degrees of freedom instead of functional of space, the generalized Wiener-Hermite functionals reduce to generalized Wiener-Hermite polynomials which are seen in the literature (Appel \& Fériet 1926). For the application to modern cosmology, the generalized Wiener-Hermite functionals are more appropriate than the others.

We expand the functionals $F_{\mathrm{m}}$ and $F_{\mathrm{g}}$ by these functionals. It is useful to define the following functional which is an infinite dimensional linear combination of $\mathcal{H}^{(n)}$ :

$$
\mathcal{H}_{(n)}\left(\boldsymbol{x}_{1}, \ldots, \boldsymbol{x}_{n}\right)=\int d^{3} y_{1} \cdots d^{3} y_{n} \xi_{\mathrm{L}}\left(\boldsymbol{x}_{1}, \boldsymbol{y}_{1}\right) \cdots \xi_{\mathrm{L}}\left(\boldsymbol{x}_{n}, \boldsymbol{y}_{n}\right) \mathcal{H}^{(n)}\left(\boldsymbol{y}_{1}, \ldots, \boldsymbol{y}_{n}\right),
$$

which we also call as generalized Wiener-Hermite functional. The explicit form of the first three generalized Wiener-Hermite functionals are

$$
\begin{aligned}
& \mathcal{H}_{(0)}=1, \\
& \mathcal{H}_{(1)}(\boldsymbol{x})=\delta_{\mathrm{L}}(\boldsymbol{x}), \\
& \mathcal{H}_{(2)}\left(\boldsymbol{x}_{1}, \boldsymbol{x}_{2}\right)=\delta_{\mathrm{L}}\left(\boldsymbol{x}_{1}\right) \delta_{\mathrm{L}}\left(\boldsymbol{x}_{2}\right)-\xi_{\mathrm{L}}\left(\boldsymbol{x}_{1}-\boldsymbol{x}_{2}\right), \\
& \mathcal{H}_{(3)}\left(\boldsymbol{x}_{1}, \boldsymbol{x}_{2}, \boldsymbol{x}_{3}\right)=\delta_{\mathrm{L}}\left(\boldsymbol{x}_{1}\right) \delta_{\mathrm{L}}\left(\boldsymbol{x}_{2}\right) \delta_{\mathrm{L}}\left(\boldsymbol{x}_{3}\right) \\
& \quad-\left[\xi_{\mathrm{L}}\left(\boldsymbol{x}_{1}-\boldsymbol{x}_{2}\right) \delta_{\mathrm{L}}\left(\boldsymbol{x}_{3}\right)+\xi_{\mathrm{L}}\left(\boldsymbol{x}_{2}-\boldsymbol{x}_{3}\right) \delta_{\mathrm{L}}\left(\boldsymbol{x}_{1}\right)+\xi_{\mathrm{L}}\left(\boldsymbol{x}_{3}-\boldsymbol{x}_{1}\right) \delta_{\mathrm{L}}\left(\boldsymbol{x}_{2}\right)\right] .
\end{aligned}
$$

The generalized Wiener-Hermite functionals have the orthogonality relation:

$$
\begin{aligned}
& \left\langle\mathcal{H}_{(n)}\left(\boldsymbol{x}_{1}, \ldots, \boldsymbol{x}_{n}\right) \mathcal{H}^{(m)}\left(\boldsymbol{y}_{1}, \ldots, \boldsymbol{y}_{m}\right)\right\rangle \\
& \quad=\delta_{n m}\left[\delta^{3}\left(\boldsymbol{x}_{1}-\boldsymbol{y}_{1}\right) \cdots \delta^{3}\left(\boldsymbol{x}_{n}-\boldsymbol{y}_{n}\right)+\operatorname{sym} .\left(\boldsymbol{y}_{1}, \ldots, \boldsymbol{y}_{n}\right)\right],
\end{aligned}
$$


where sym.(...) denote the terms of symmetric permutation of the preceding term with respect to the indices. In this case, there are $n$ ! terms in total in the right hand side of equation (2.11). The Gaussian averaging $\langle\cdots\rangle$ denote the averaging by linear density field $\delta_{\mathrm{L}}$ by the random Gaussian probability functional $\mathcal{P}\left[\delta_{\mathrm{L}}\right]$ :

$$
\langle\cdots\rangle=\int\left[d \delta_{\mathrm{L}}\right] \cdots \mathcal{P}\left[\delta_{\mathrm{L}}\right]
$$

where

$$
\mathcal{P}\left[\delta_{\mathrm{L}}\right]=\mathcal{N} \exp \left[-\frac{1}{2} \int d^{3} x d^{3} y \delta_{\mathrm{L}}(\boldsymbol{x}) \xi_{\mathrm{L}}^{-1}(\boldsymbol{x}, \boldsymbol{y}) \delta_{\mathrm{L}}(\boldsymbol{y})\right] .
$$

The formal normalization constant $\mathcal{N}$ is given by

$$
\mathcal{N}=\left\{\int\left[d \delta_{\mathrm{L}}\right] \exp \left[-\frac{1}{2} \int d^{3} x d^{3} y \delta_{\mathrm{L}}(\boldsymbol{x}) \xi_{\mathrm{L}}^{-1}(\boldsymbol{x}, \boldsymbol{y}) \delta_{\mathrm{L}}(\boldsymbol{y})\right]\right\}^{-1}
$$

Because the degrees of freedom is infinite, $\mathcal{N}^{-1}$ formally diverges, but the proper regularization is always possible by discretizing the three dimensional continuum space. One can prove the orthogonality equation (2.11), just generalizing the proof of orthogonality of simple Hermite polynomial, which is well known.

We consider the functionals of equation (2.5) as base functionals for the expansion of the functional of mass density field, and of galaxy density field:

$$
\delta_{\mathrm{A}}\left(\boldsymbol{r},\left[\delta_{\mathrm{L}}\right]\right)=\sum_{n=1}^{\infty} \frac{1}{n !} \int d^{3} x_{1} \cdots d^{3} x_{n} K_{\mathrm{A}}^{(n)}\left(\boldsymbol{r}-\boldsymbol{x}_{1}, \cdots, \boldsymbol{r}-\boldsymbol{x}_{n}\right) \mathcal{H}_{(n)}\left(\boldsymbol{x}_{1}, \ldots, \boldsymbol{x}_{n}\right),
$$

where $\mathrm{A}=\mathrm{m}$ or $\mathrm{g}$. The reason why $n=0$ term is not appeared in the above expression is that $\left\langle\delta_{\mathrm{A}}\right\rangle=0$ and $\left\langle\mathcal{H}_{(n)}\right\rangle=0$ for $n>0$ [set $m=0$ in equation (2.11)]. This expansion is complete because the kernel $K^{(n)}$ is uniquely given by

$$
\begin{aligned}
K_{\mathrm{A}}^{(n)}\left(\boldsymbol{r}-\boldsymbol{x}_{1}, \ldots, \boldsymbol{r}-\boldsymbol{x}_{n}\right) & =\left\langle\mathcal{H}^{(n)}\left(\boldsymbol{x}_{1}, \ldots, \boldsymbol{x}_{n}\right) \delta_{\mathrm{A}}\left(\boldsymbol{r},\left[\delta_{\mathrm{L}}\right]\right)\right\rangle \\
& =\left\langle\frac{\delta^{n} \delta_{\mathrm{A}}\left(\boldsymbol{r},\left[\delta_{\mathrm{L}}\right]\right)}{\delta \delta_{\mathrm{L}}\left(\boldsymbol{x}_{1}\right) \cdots \delta \delta_{\mathrm{L}}\left(\boldsymbol{x}_{n}\right)}\right\rangle .
\end{aligned}
$$

According to the expansion (2.15) and the orthogonality relation (2.11), the two-point auto-correlation function of matter $\xi_{\mathrm{mm}}$ and of galaxies $\xi_{\mathrm{gg}}$ and the cross correlation function $\xi_{\mathrm{mg}}$ are given by

$$
\begin{aligned}
\xi_{\mathrm{AB}}(\boldsymbol{r})= & \left\langle\delta_{\mathrm{A}}\left(\boldsymbol{r}_{1}\right) \delta_{\mathrm{B}}\left(\boldsymbol{r}_{2}\right)\right\rangle \\
= & \sum_{n=1}^{\infty} \frac{1}{n !} \int d^{3} x_{1} \cdots d^{3} x_{n} d^{3} y_{1} \cdots d^{3} y_{n} K_{\mathrm{A}}^{(n)}\left(\boldsymbol{x}_{1}, \ldots, \boldsymbol{x}_{n}\right) K_{\mathrm{B}}^{(n)}\left(\boldsymbol{y}_{1}, \ldots, \boldsymbol{y}_{n}\right) \\
& \quad \times \xi_{\mathrm{L}}\left(\boldsymbol{r}+\boldsymbol{x}_{1}+\boldsymbol{y}_{1}\right) \xi_{\mathrm{L}}\left(\boldsymbol{r}+\boldsymbol{x}_{2}+\boldsymbol{y}_{2}\right) \cdots \xi_{\mathrm{L}}\left(\boldsymbol{r}+\boldsymbol{x}_{n}+\boldsymbol{y}_{n}\right),
\end{aligned}
$$


where $\mathrm{A}, \mathrm{B}=\mathrm{m}, \mathrm{g}$, and $\boldsymbol{r}_{1}-\boldsymbol{r}_{2}=\boldsymbol{r}$. Note that this expansion is valid as long as $\delta_{\mathrm{L}}$ is a random Gaussian field. If the initial density field is non-Gaussian, there are additional terms in equation (2.18) which depend on initial higher-order correlation functions [see Matsubara (1995) for detail].

Both the nonlocal kernels $K_{\mathrm{m}}$ and $K_{\mathrm{g}}$ do not depend on $\boldsymbol{r}$. Thus, on large scales with respect to the separation $\boldsymbol{r}$, we can approximate equation (2.18) by only considering lower order terms of $\xi_{\mathrm{L}}$, provided that the kernel $K_{\mathrm{A}}^{(n)}$ does not have broad profile.

If the kernel falls off slowly on large scales, we can not truncate the expansion (2.18). In the rest of this paper, we assume the lowest order term in the expansion (2.18) actually dominates the higher-order terms. Before proceeding to the analysis of the lowest order approximation, we consider the cases in which this assumption breaks down. Imagine, for example, that $\xi_{\mathrm{L}}(r) \sim r^{-\gamma}$, and $K_{\mathrm{A}}^{(n)}\left(\boldsymbol{x}_{1}, \ldots, \boldsymbol{x}_{n}\right) \sim\left(x_{1} \cdots x_{n}\right)^{-s_{\mathrm{A}}}$ on large scales. Then the $n$-th order term in the integral in expansion (2.18) is approximately given by

$$
\begin{gathered}
\int \frac{d^{3} x_{1} \cdots d^{3} x_{n} d^{3} y_{1} \cdots d^{3} y_{n}}{\left(x_{1} \cdots x_{n}\right)^{s_{\mathrm{A}}}\left(y_{1} \cdots y_{n}\right)^{s_{\mathrm{B}}}\left|\boldsymbol{r}+\boldsymbol{x}_{1}+\boldsymbol{y}_{1}\right|^{\gamma} \cdots\left|\boldsymbol{r}+\boldsymbol{x}_{n}+\boldsymbol{y}_{n}\right|^{\gamma}} \\
=\left(\int \frac{d^{3} x d^{3} y}{x^{s_{\mathrm{A}}} y^{s_{\mathrm{B}}}|\boldsymbol{r}+\boldsymbol{x}+\boldsymbol{y}|^{\gamma}}\right)^{n} \propto\left(r^{6-s_{\mathrm{A}}-s_{\mathrm{B}}-\gamma}\right)^{n} .
\end{gathered}
$$

The last expression is derived by the fact that the integral is the form of convolution and the (3D) Fourier transform of $k^{n}$ is proportional to $r^{-n-3}$ (Peebles 1980). As seen by this expression, $6-s_{\mathrm{A}}-s_{\mathrm{B}}-\gamma$ should be negative to ensure the equation (2.19) actually falls off on large scales. For example, if $K_{\mathrm{g}}^{(2)}$ falls off as $x_{1}^{-2} x_{2}^{-2}$, and $\gamma \leq 2$, then equation (2.19) does not fall off anymore. On the other hand, if $s_{\mathrm{A}, \mathrm{B}} \geq 3$, then equation (2.19) falls off rapidly enough so that the higher order terms can be neglected. In the latter case, the spatial integration of $K^{(n)}$ is finite. It is a natural assumption that the spatial integration of $K^{(n)}$ is finite. If it is infinite, as seen from equation (2.15), the information of density fluctuations of infinitely distant places affect the galaxy formation as much as, or more than the fluctuations of nearer places, which is unlikely in reality. We assume $s_{\mathrm{A}, \mathrm{B}} \geq 3$ in the rest of this paper. In other words, we assume that Fourier transform of higher-order kernels $\widetilde{K}_{\mathrm{A}}^{(n)}\left(\boldsymbol{k}_{1}, \ldots, \boldsymbol{k}_{n}\right)$ are finite in the limit $k_{1}, \ldots, k_{n} \rightarrow 0$.

Note that the expansion (2.18) is essentially different from usual perturbative approach by Taylor expansion of density contrast itself. Instead, we employ the orthogonal expansion for galaxy and mass density fields, and the resulting expression, (2.18), can be interpreted as an asymptotic expansion by correlation function, $\xi_{\mathrm{L}}$. Thus, we only assume the smallness of correlation function $\xi_{\mathrm{L}}$ on large scales, whether the density contrast on small scale is large or not. 


\subsection{A Sample Calculation of Kernels}

Since our argument in this section is extremely formal, it would be instructive to calculate an example. Consider a model in which $\delta_{\mathrm{g}}=\delta_{\mathrm{m}}+\delta_{\mathrm{m}}^{2}$ and $\delta_{\mathrm{m}}$ is calculated up to second order in perturbation theory [e.g., Fry (1984)]:

$$
\delta_{\mathrm{m}}(\boldsymbol{r})=\delta_{\mathrm{L}}(\boldsymbol{r})+\int d^{3} x_{1} d^{3} x_{2} R\left(\boldsymbol{r}-\boldsymbol{x}_{1}, \boldsymbol{r}-\boldsymbol{x}_{2}\right) \delta_{\mathrm{L}}\left(\boldsymbol{x}_{1}\right) \delta_{\mathrm{L}}\left(\boldsymbol{x}_{2}\right)
$$

where

$$
R\left(\boldsymbol{x}_{1}, \boldsymbol{x}_{2}\right)=\int \frac{d^{3} k_{1}}{(2 \pi)^{3}} \frac{d^{3} k_{2}}{(2 \pi)^{3}}\left[\frac{5}{7}+\frac{\boldsymbol{k}_{1} \cdot \boldsymbol{k}_{2}}{2 k_{1} k_{2}}\left(\frac{k_{1}}{k_{2}}+\frac{k_{2}}{k_{1}}\right)+\frac{2}{7}\left(\frac{\boldsymbol{k}_{1} \cdot \boldsymbol{k}_{2}}{k_{1} k_{2}}\right)^{2}\right] e^{i \boldsymbol{k}_{1} \cdot \boldsymbol{x}_{1}+i \boldsymbol{k}_{2} \cdot \boldsymbol{x}_{2}}
$$

and we assume the Einstein-de Sitter Universe for simplicity. Then, according to equation (2.17), we can calculate the kernels as

$$
\begin{aligned}
K_{\mathrm{m}}^{(1)}(\boldsymbol{x})=\delta^{3}(\boldsymbol{x}) & \\
K_{\mathrm{m}}^{(2)}\left(\boldsymbol{x}_{1}, \boldsymbol{x}_{2}\right)= & 2 R\left(\boldsymbol{x}_{1}, \boldsymbol{x}_{2}\right), \\
K_{\mathrm{g}}^{(1)}(\boldsymbol{x})=\delta^{3}(\boldsymbol{x})+2 \int d^{3} y R(\boldsymbol{x}, \boldsymbol{y}) \xi_{\mathrm{L}}(\boldsymbol{y}) & \\
& +2 \delta^{3}(\boldsymbol{x}) \int d^{3} y_{1} d^{3} y_{2} R\left(\boldsymbol{y}_{1}, \boldsymbol{y}_{2}\right) \xi_{\mathrm{L}}\left(\boldsymbol{y}_{1}-\boldsymbol{y}_{2}\right), \\
K_{\mathrm{g}}^{(2)}\left(\boldsymbol{x}_{1}, \boldsymbol{x}_{2}\right)= & 2 \delta^{3}\left(\boldsymbol{x}_{1}\right) \delta^{3}\left(\boldsymbol{x}_{2}\right)+2 R\left(\boldsymbol{x}_{1}, \boldsymbol{x}_{2}\right) \\
& +4 R\left(\boldsymbol{x}_{1}, \boldsymbol{x}_{2}\right) \int d^{3} y_{1} d^{3} y_{2} R\left(\boldsymbol{y}_{1}, \boldsymbol{y}_{2}\right) \xi_{\mathrm{L}}\left(\boldsymbol{y}_{1}-\boldsymbol{y}_{2}\right) \\
& +8 \int d^{3} y_{1} d^{3} y_{2} R\left(\boldsymbol{x}_{1}, \boldsymbol{y}_{1}\right) R\left(\boldsymbol{x}_{2}, \boldsymbol{y}_{2}\right) \xi_{\mathrm{L}}\left(\boldsymbol{y}_{1}-\boldsymbol{y}_{2}\right) .
\end{aligned}
$$

Since $R\left(\boldsymbol{x}_{1}, \boldsymbol{x}_{2}\right)$ drops off as $\left|\boldsymbol{x}_{1}\right|^{-3}\left|\boldsymbol{x}_{2}\right|^{-3}$ on large scales, the discussion at the end of the previous subsection suggests that $n$-th order term in equation (2.18) actually drops off as $\left(\xi_{\mathrm{L}}\right)^{n}$ on large scales.

\subsection{Stochastic Parameters in Linear Regime}

In the following, we are interested in large scales and consider only the lowest order approximation of equation (2.18):

$$
\xi_{\mathrm{AB}}(\boldsymbol{r})=\int d^{3} x d^{3} y K_{\mathrm{A}}^{(1)}(\boldsymbol{x}) K_{\mathrm{B}}^{(1)}(\boldsymbol{y}) \xi_{\mathrm{L}}(\boldsymbol{r}+\boldsymbol{x}+\boldsymbol{y}),
$$

assuming that this term does not vanish and that the higher-order terms are negligible. Since this expression has the form of convolution, it becomes just products in Fourier space. 
From statistical isotropy, the Fourier transform of $K^{(1)}$, denoted as $\widetilde{K}^{(1)}$, is a function of the absolute value of the wave vector $k=|\boldsymbol{k}|$ :

$$
P_{\mathrm{AB}}(k)=\widetilde{K}_{\mathrm{A}}^{(1)}(k) \widetilde{K}_{\mathrm{B}}^{(1)}(k) P_{\mathrm{L}}(k),
$$

where $P_{\mathrm{L}}(k)$ is the linear power spectrum円.

The linear-scale power spectrum of mass is simply given by $P_{\mathrm{mm}}(k)=P_{\mathrm{L}}(k)$. This means $\widetilde{K}_{\mathrm{m}}^{(1)}(k)=1$ on linear scales. Thus, denoting $b_{\mathrm{F}}(k)=\widetilde{K}_{\mathrm{g}}^{(1)}(k)$,

$$
\begin{aligned}
& P_{\mathrm{mm}}(k)=P_{\mathrm{L}}(k), \\
& P_{\mathrm{mg}}(k)=b_{\mathrm{F}}(k) P_{\mathrm{L}}(k), \\
& P_{\mathrm{gg}}(k)=b_{\mathrm{F}}^{2}(k) P_{\mathrm{L}}(k) .
\end{aligned}
$$

These equations are valid as long as $\widetilde{K}_{\mathrm{g}}^{(1)}(k) \neq 0$. If $\widetilde{K}_{\mathrm{g}}^{(1)}(k)=0$, equation $(2.30)$ vanishes and is no longer the lowest order in the expansion of equation (2.18). In such case, we should consider the higher-order terms.

In equations (2.31)-(2.33), $b_{\mathrm{F}}(k)$ can be identified to the linear bias parameter in Fourier space, and is given by, from Fourier transform of equation (2.17),

$$
b_{\mathrm{F}}(k)=\int d^{3} x e^{i \boldsymbol{k} \cdot \boldsymbol{x}}\left\langle\frac{\delta \delta_{\mathrm{g}}(\mathbf{0})}{\delta \delta_{\mathrm{L}}(\boldsymbol{x})}\right\rangle=\left\langle\frac{\delta \delta_{\mathrm{g}}(\mathbf{0})}{\delta \widetilde{\delta}_{\mathrm{L}}(\boldsymbol{k})}\right\rangle,
$$

where $\widetilde{\delta}_{\mathrm{L}}(\boldsymbol{k})$ is a Fourier transform of linear density fluctuations, $\delta_{\mathrm{L}}(\boldsymbol{x})$.

These simple equations (2.31) $-(2.34)$ are the primary results of this paper. These equations show, as long as $\widetilde{K_{\mathrm{g}}^{(1)}}(k) \neq 0$, that there are no residual cross correlation in Fourier space in linear regime (except for the constant term which comes from the small scale behavior of correlation function) and that the bias parameter in Fourier space is $b_{\mathrm{F}}(k)$ which is scale-dependent and is related to nonlinearity and nonlocality of galaxy formation through equation (2.34). This means that the Fourier-mode stochasticity which arise from the nonlinearity and nonlocality of the galaxy formation vanishes in linear regime. Thus, the cross correlation $r(k)$ in Fourier space should approach to unity in large-scale limit, as long as the galaxy formation is such that $\widetilde{K}_{\mathrm{g}}^{(1)}(k) \neq 0$.

The case, $\widetilde{K}_{\mathrm{g}}^{(1)}(k)=0$ can happen in special cases. It can happen, for example, when the large-scale linear power is completely erased by some peculiar form of nonlocal biasing,

\footnotetext{
${ }^{1}$ If we consider $P_{\mathrm{AB}}(k)$ as the true spectrum, there is possibly a constant term in addition to the above equation, which comes from the small scale inaccuracy of expression (2.29) [see Scherrer \& Weinberg (1998); Dekel \& Lahav (1998)]. However, in the following, we consider $P_{\mathrm{AB}}(k)$ as a merely mathematical quantity which represents just the Fourier transform of $\xi_{\mathrm{AB}}(r)$ of the equation (2.29).
} 
and mode-mode coupling from nonlinear scales dominates on linear scales. It also can happen when the biasing is represented by an even function of $\delta_{\mathrm{L}}$, e.g., purely quadratic, $\delta_{\mathrm{g}}=\delta_{\mathrm{L}}^{2}-\left\langle\delta_{\mathrm{L}}^{2}\right\rangle$, or quartic, $\delta_{\mathrm{g}}=\delta_{\mathrm{L}}^{4}-\left\langle\delta_{\mathrm{L}}^{4}\right\rangle$, etc. We assume the galaxy formation does not have such special form and satisfies the condition $\widetilde{K}_{\mathrm{g}}^{(1)}(k) \neq 0$ in the rest of this paper.

The vanishing stochasticity is an important constraint on large scales. At this point, the naive introduction of stochasticity $r$ in linear or quasi-linear regime, as is sometimes done in literatures, should be cautious. On large scales, $r$ could not be freely adjusted, but would be close to unity. This fact is already noticed in the paper by Dekel \& Lahav (1998), in which only a specific, simple model is considered. The same conclusion is derived by Scherrer \& Weinberg (1998) based on local galaxy formation scheme. Our conclusions apply not only to local schemes, but also to nonlocal schemes of galaxy formation. The difference between local and nonlocal scheme is that local bias generates constant bias factor, while nonlocal bias generates scale-dependent one.

If there is a non-unity value of $r$ in large-scale limit, it means that there is some exotic stochasticity which is not relevant to the initial density fluctuations, or that $\widetilde{K}_{\mathrm{g}}^{(1)}(k)=0$. As all the structures in the universe are supposed to be formed from initial density fluctuations, there is no specific reason to introduce such kind of exotic processes, at least in the framework of the present standard theory of structure formation in the universe.

Of course, in nonlinear regime where the approximation of equation (2.29) breaks down, the stochasticity in Fourier space arises by mode-mode coupling. Since the dynamics of such nonlinear regime is complex enough to trace analytically, the concept of stochastic bias is useful especially in this regime. In the nonlinear regime, one should be aware that the nonlinearity of galaxy-density relation also dominate and that only parameters $b$ and $r$ are not sufficient to characterize the biasing properties (Dekel \& Lahav 1998).

The equations (2.31)-(2.33) is valid on scales larger than a nonlinear scale $2 \pi / k_{\mathrm{NL}}$, because these equations are derived from lowest order approximation, which assumes $k^{3} P_{\mathrm{L}}(k)$ is small. If the nonlocality of galaxy formation is small and $K_{\mathrm{A}}^{(1)}(\boldsymbol{x})$ is localized on some scales smaller than $2 \pi / k_{\mathrm{g}}$, then its Fourier transform, $b_{\mathrm{F}}(k)$, will be constant for $k<k_{\mathrm{g}}$. If $k_{\mathrm{g}}>k_{\mathrm{NL}}$, the bias factor is constant in the validity region of the equations. This is equivalent to the purely local galaxy formation. In contrast, if $k_{\mathrm{g}}<k_{\mathrm{NL}}$, there appears the scale-dependence of bias parameter besides mode-mode coupling. This scale-dependence comes from the nonlocality of the galaxy formation. The $k$-dependence of $b_{\mathrm{F}}(k)$ thus is not negligible on scales below $2 \pi / k_{\mathrm{g}}$ and its behavior can be describable by equation (2.34) on scales above $2 \pi / k_{\mathrm{NL}}$.

Even when there is no stochasticity in Fourier space, it may still appear in real space 
on linear scales because of the scale-dependence of bias parameter, besides mode-mode coupling. Actually, the galaxy-density cross correlation and galaxy correlation for a smoothed field with linear smoothing length $R$ are given by

$$
\begin{aligned}
& \left\langle\delta_{\mathrm{m}}(\boldsymbol{x} ; R) \delta_{\mathrm{g}}(\boldsymbol{x} ; R)\right\rangle=\int \frac{k^{2} d k}{2 \pi^{2}} P_{\mathrm{mg}}(k) W^{2}(k R)=\int \frac{k^{2} d k}{2 \pi^{2}} b_{\mathrm{F}}(k) P_{\mathrm{L}}(k) W^{2}(k R), \\
& \left\langle\delta_{\mathrm{g}}(\boldsymbol{x} ; R)^{2}\right\rangle=\int \frac{k^{2} d k}{2 \pi^{2}} P_{\mathrm{gg}}(k) W^{2}(k R)=\int \frac{k^{2} d k}{2 \pi^{2}} b_{\mathrm{F}}^{2}(k) P_{\mathrm{L}}(k) W^{2}(k R),
\end{aligned}
$$

where $W$ is a Fourier transform of a smoothing function. This expression and equation (1.2) explicitly show the scale dependence and stochasticity of the biasing in real space. We define the following notation for k-space averaging for an arbitrary function $h(k)$ :

$$
\bar{h}(R)=\frac{\int \frac{k^{2} d k}{2 \pi^{2}} h(k) P_{\mathrm{L}}(k) W^{2}(k R)}{\int \frac{k^{2} d k}{2 \pi^{2}} P_{\mathrm{L}}(k) W^{2}(k R)} .
$$

Then, equation (1.2) reduces to, simply,

$$
b(R)=\sqrt{\overline{b_{\mathrm{F}}^{2}}(R)}, \quad r(R)=\frac{\overline{b_{\mathrm{F}}}(R)}{\sqrt{\overline{b_{\mathrm{F}}^{2}}(R)}}
$$

To obtain more insight on this scale-dependence and stochasticity, let us expand the bias parameter $b_{\mathrm{F}}(k)$ in terms of $\left(k / k_{\mathrm{g}}\right)^{2}$, where $2 \pi / k_{\mathrm{g}}$ is the scale of nonlocality of galaxy formation as above (terms of odd power of $k$ do not appear for reflection symmetry):

$$
b_{\mathrm{F}}(k)=b_{\mathrm{F}}^{(0)}+b_{\mathrm{F}}^{(1)}\left(k / k_{\mathrm{g}}\right)^{2}+\frac{b_{\mathrm{F}}^{(2)}}{2 !}\left(k / k_{\mathrm{g}}\right)^{4}+\mathcal{O}\left(k / k_{\mathrm{g}}\right)^{6} .
$$

With this expansion, equation (2.38) is expanded in a straightforward manner, and the results are

$$
\begin{aligned}
b(R)= & b_{\mathrm{F}}^{(0)}+3 \gamma^{2} b_{\mathrm{F}}^{(1)}\left(R_{*} k_{\mathrm{g}}\right)^{-2} \\
& +\frac{9 \gamma^{2}}{2 b_{\mathrm{F}}^{(0)}}\left[b_{\mathrm{F}}^{(0)} b_{\mathrm{F}}^{(2)}+\left(1-\gamma^{2}\right)\left(b_{\mathrm{F}}^{(1)}\right)^{2}\right]\left(R_{*} k_{\mathrm{g}}\right)^{-4}+\mathcal{O}\left(R_{*} k_{\mathrm{g}}\right)^{-6}, \\
r(R)=1 & -\frac{9}{2} \gamma^{2}\left(1-\gamma^{2}\right)\left(\frac{b_{\mathrm{F}}^{(1)}}{b_{\mathrm{F}}^{(0)}}\right)^{2}\left(R_{*} k_{\mathrm{g}}\right)^{-4}+\mathcal{O}\left(R_{*} k_{\mathrm{g}}\right)^{-6}
\end{aligned}
$$

In this expression, spectral parameters $\gamma$ and $R_{*}$ (Bardeen et al. 1986, BBKS, hereafter) are given by

$$
\gamma=\frac{\overline{k^{2}}(R)}{\sqrt{k^{4}}(R)}, \quad R_{*}=\sqrt{\frac{3 \overline{k^{2}}(R)}{\overline{k^{4}}(R)}}
$$


where notation of the equation (2.37) is applied. The parameter $\gamma$ is of order unity, and $R_{*}$ is of order $R$. For example, if the power spectrum has the form of power-law, $P_{\mathrm{L}}(k) \propto k^{n}$, and the smoothing function is Gaussian, $W(x) \propto \exp \left(-x^{2} / 2\right)$, these parameters are given by $\gamma^{2}=(n+3) /(n+5)$ and $R_{*}=(6 /(n+5))^{1 / 2} R$.

Equations (2.40) and (2.41) give the expression for stochastic bias parameter from nonlocality of galaxy formation. These equations represent the minimal stochasticity which is inevitable when bias is scale dependent. We have neglected the mode coupling near nonlinear scales. The scale-dependence and stochasticity appears on the scale of the nonlocality, $2 \pi / k_{\mathrm{g}}$. On scales larger than nonlocality, such scale-dependence and stochasticity disappears. Especially, stochasticity rapidly vanishes for the lack of $R^{-2}$ term in equation (2.41). These results do not depend on specific details of galaxy formation as long as $\widetilde{K}_{\mathrm{g}}^{(1)}(k) \neq 0$, and higher-order terms are negligible. The information of galaxy formation involves only through two parameters, $b_{\mathrm{F}}^{(0)}$ and $b_{\mathrm{F}}^{(1)}$, up to the order $\left(k_{\mathrm{g}} R\right)^{-2}$, or three parameters $b_{\mathrm{F}}^{(0)}, b_{\mathrm{F}}^{(1)}$ and $b_{\mathrm{F}}^{(2)}$ up to the order $\left(k_{\mathrm{g}} R\right)^{-4}$. These parameters are related to galaxy formation through equation (2.34) which should be calculable if we could know the details of galaxy formation process. Otherwise, they can be considered as free parameters to be fitted by observation, instead of fitting functions $b(R)$ and $r(R)$, which have the infinite degrees of freedom.

\section{SIMPLE MODELS OF NONLOCAL GALAXY FORMATION}

So far the argument is quite formal. In this section, we consider specific models of galaxy formation, i.e., the Lagrangian local biasing models, the cooperative model, and the peak model, as simple examples. Although the quantitative correspondence of these models and the actual galaxy formation still needs investigation, these examples can give qualitative aspects on the nonlocal galaxy formation.

\subsection{Lagrangian Local Biasing Models}

In the Lagrangian local galaxy formation models, the number density of galaxies is a local function of a smoothed linear density field. The smoothed density field, however, is a nonlocal function of linear density field, $\delta_{\mathrm{L}}$. Thus, in some sense, local galaxy formation models fall in the category of nonlocal models, with particularly simple functional form:

$$
\rho_{\mathrm{g}}(\boldsymbol{r})=f\left(\delta_{\mathrm{Ls}}(\boldsymbol{r})\right)
$$


where $f$ is an usual one-variable function of the smoothed linear density field. The smoothed linear density field, $\delta_{\mathrm{Ls}}$, is defined by

$$
\delta_{\mathrm{L} s}(\boldsymbol{r})=\int d^{3} x W_{\mathrm{s}}^{(\mathrm{real})}(\boldsymbol{r}-\boldsymbol{x}) \delta_{\mathrm{L}}(\boldsymbol{x}),
$$

where $W_{\mathrm{s}}^{(\mathrm{real})}$ is a smoothing kernel function with smoothing length $s$. With this particularly simple form, the bias parameter in Fourier space, given by equation (2.34), reduces to

$$
b_{\mathrm{F}}(k)=\frac{\left\langle f^{\prime}\left(\delta_{\mathrm{Ls}}\right)\right\rangle}{\left\langle f\left(\delta_{\mathrm{L} s}\right)\right\rangle} W_{\mathrm{s}}(k s)
$$

where $W_{\mathrm{s}}$ is a Fourier transform of the smoothing kernel $W_{\mathrm{s}}^{(\text {real })}$. All the other higher-order kernels $K^{(n)}(n \geq 2)$ vanish. This result is essentially the same which is derived previously in real space (Szalay 1988; Coles 1993):

$$
\xi_{\mathrm{g}}(r)=\left(\frac{\left\langle f\left(\delta_{\mathrm{L} s}\right) H_{1}\left(\delta_{\mathrm{L} s} / \sigma_{\mathrm{L} s}\right)\right\rangle}{\left\langle f\left(\delta_{\mathrm{L} s}\right)\right\rangle}\right)^{2} \frac{\xi(r)}{\sigma_{\mathrm{L} s}^{2}},
$$

since $\left\langle f\left(\delta_{\mathrm{L} s}\right) H_{1}\left(\delta_{\mathrm{L} s} / \sigma_{\mathrm{L} s}\right)\right\rangle=\sigma_{\mathrm{L} s}\left\langle f^{\prime}\left(\delta_{\mathrm{L} s}\right)\right\rangle$.

In usual nonlocal biasing models, the smoothing length $s$ is taken to be in nonlinear regime. As we consider linear scales, $W_{\mathrm{s}}(k s) \sim 1$ and there is no scale-dependence on $b_{\mathrm{F}}(k)$ and thus there is no stochasticity in real space. This result is consistent with the work by Scherrer \& Weinberg (1998), who showed the local models produce constant bias factor and there is negligible stochasticity on large scales. Examples of local models are density-threshold bias, $f(x) \propto \theta\left(x-\nu \sigma_{\mathrm{Ls}}\right)$, where $\theta(x)$ is a step function (Kaiser 1984; Jensen \& Szalay 1986), weighted bias, $f(x) \propto(1+x) \theta\left(x-\nu \sigma_{L s}\right)$ (Catelan et al. 1994), Cen-Ostriker bias, $f(x) \propto \exp \left\{C_{0}+C_{1} \ln (1+x)+C_{2}[\ln (1+x)]^{2}\right\}$ (Cen \& Ostriker 1992), etc. In nonlinear regime, there is really scale-dependence on $b_{\mathrm{F}}(k)$ (Mann, Peacock, \& Heavens 1998; Narayanan, Berlind, \& Weinberg 1998)]2.

Recently, the Mo \& White model (Mo \& White 1996) for the clustering of dark matter halos, which is an extension of Press-Schechter formalism (Press \& Schechter 1974; Cole \& Kaiser 1989; Bond et al. 1991), is interested in (e.g., Catelan et al. 1998; Catelan, Matarrese \& Porciani 1998; Jing 1998; Sheth \& Lemson 1998). This model is also an example of the Lagrangian local biasing model, because the halo density contrast $\delta_{\mathrm{h}}(1 \mid 0)$ in their model is determined by linear density field $\delta_{0}$ which is smoothed on scale $R_{0}$.

\footnotetext{
${ }^{2}$ Their work is based on numerical simulation and Eulerian local biasing, in which the biasing function is a local function of nonlinear density field. Thus, strictly speaking, their analyses are for Lagrangian nonlocal biasing scheme in the terminology of this paper. However, the difference between Lagrangian and Eulerian correlation functions is negligible (higher-order) on linear scales.
} 


\subsection{Cooperative Model}

Next, we consider cooperative galaxy formation model introduced by Bower et al. (1993), in which they showed that a large-scale $\left(\sim 20 h^{-1} \mathrm{Mpc}\right)$, but weak modulation of galaxy luminosities can reconcile the discrepancy between the SCDM power spectrum and APM galaxy data (see also Babul \& White 1991). As a simple example, they identify sites for galaxy formation as places where density contrast $\delta$ satisfies the following relation

$$
\delta(\boldsymbol{r})>\nu \sigma-\kappa \bar{\delta}(\boldsymbol{r})
$$

where $\bar{\delta}$ is the density contrast smoothed on a scale $R_{\text {mod }}$, which represent the large-scale modulation, and $\kappa$ is a constant which is called as "the modulation coefficient". This simple model is mathematically equivalent to the standard density-threshold bias model, but for the new field defined by

$$
\delta^{\prime}(\boldsymbol{r})=\delta(\boldsymbol{r})+\kappa \bar{\delta}(\boldsymbol{r})
$$

It is easy to see that this new field is just a new density field with a smoothing function $W_{\mathrm{s}}(k s)+\kappa W_{\text {mod }}\left(k R_{\text {mod }}\right)$, where $W_{\mathrm{s}}$ and $W_{\text {mod }}$ are smoothing function for $\delta$ and $\bar{\delta}$, respectively. The bias parameter for the cooperative model in Fourier space is similar to equation (3.3):

$$
\begin{aligned}
b_{\mathrm{F}}(k) & =\frac{\left\langle d \theta\left(\delta^{\prime}-\nu \sigma\right) / d \delta^{\prime}\right\rangle}{\left\langle\theta\left(\delta^{\prime}-\nu \sigma\right)\right\rangle}\left[W_{\mathrm{s}}(k s)+\kappa W_{\text {mod }}\left(k R_{\text {mod }}\right)\right] \\
& =\sqrt{\frac{2}{\pi}} \frac{e^{-\nu^{\prime 2} / 2}}{\operatorname{erfc}\left(\nu^{\prime} / \sqrt{2}\right)}\left[W_{\mathrm{s}}(k s)+\kappa W_{\text {mod }}\left(k R_{\text {mod }}\right)\right],
\end{aligned}
$$

where $\nu^{\prime}=\nu \sigma / \sqrt{\left\langle\delta^{\prime 2}\right\rangle}$. This expression and equation (2.38) enable us to evaluate the stochastic parameters. In Figure 1, we plot the scale dependence of stochastic parameters of the cooperative model. In this Figure, we assume a CDM power spectrum of BBKS

$$
\begin{gathered}
P(k)=A k^{n}\left(\frac{\ln (1+2.34 q)}{2.34 q}\right)^{2}\left[1+3.89 q+(16.1 q)^{2}+(5.46 q)^{3}+(6.71 q)^{4}\right]^{-1 / 2}, \\
q=\frac{k}{\Gamma h \mathrm{Mpc}^{-1}},
\end{gathered}
$$

with primordial spectral index $n=1$ and shape parameter $\Gamma=0.5$ and linear amplitude $A=3.35 \times 10^{5}\left(h^{-1} \mathrm{Mpc}\right)^{4}$ which corresponds to $\sigma_{8}=1$. The top-hat smoothing function is applied for smoothing $R$, while the Gaussian smoothing function is applied for smoothing $s$ and $R_{\text {mod }}$ :

$$
W(x)=\frac{3}{x^{3}}(\sin x-x \cos x), \quad W_{\mathrm{s}}(x)=W_{\bmod }(x)=e^{-x^{2} / 2},
$$


and we set $\nu=2.8, s=0.5 h^{-1} \mathrm{Mpc}$ and $R_{\bmod }=10,20,30 h^{-1} \mathrm{Mpc}$. The modulation coefficient is adjusted so as to produce the $2.5 \% \mathrm{rms}$ modulation of the threshold, according to Bower et al. (1993), i.e., $\kappa \bar{\sigma} / \nu \sigma \equiv \Delta \nu / \nu=0.025$. This required taking $\kappa=0.89,2.32$, 4.35 for $R_{\text {mod }}=10,20,30 h^{-1} \mathrm{Mpc}$, respectively円.

As one can see from the Figure, there appears the scale-dependence of bias parameter $b(R)$ on scales of $R_{\text {mod }}$ so that the galaxy clustering on large scales are enhanced by cooperative bias. This fact is a main motivation for Bower et al. (1993) to introduce the cooperative model. The stochastic parameter $r(R)$ is very close to unity except on the modulation scale, where there is weak stochasticity due to scale dependence of bias parameter.

\subsection{Peak Model}

Next example of nonlocal galaxy formation is the peak model. In the peak model, the sites for galaxy formation are identified as high peaks of initial density field with a fixed smoothing length (see BBKS). Treating the constraint properly for density peaks is difficult but there are several approximations. In this paper, we approximate the density peaks by density extrema (Otto, Politzer \& Wise 1986; Cline et al. 1987; Catelan et al. 1988). The number density of density extrema above threshold $\delta_{\mathrm{t}}$ is given by

$$
\rho_{\mathrm{g}}(\boldsymbol{r})=\theta\left(\delta_{\mathrm{Ls}}(\boldsymbol{r})-\delta_{\mathrm{t}}\right) \delta^{3}\left(\partial_{i} \delta_{\mathrm{Ls}}(\boldsymbol{r})\right)(-1)^{3} \operatorname{det}\left(\partial_{i} \partial_{j} \delta_{\mathrm{Ls}}(\boldsymbol{r})\right)
$$

where $\delta_{\mathrm{Ls}}(\boldsymbol{r})$ is a smoothed linear density field with smoothing length $s$. Density extrema are identical to density peaks above some moderate threshold where almost all density extrema would be density peaks.

From the general consideration in the previous section, it is straightforward to obtain parameters $b(R)$ and $r(R)$ of stochastic bias for this model. From equation (2.34) and after tedious calculationf, we obtain

$$
b_{\mathrm{F}}(k)=\frac{1}{\sigma_{\mathrm{s} 0} H_{2}(\nu)}\left[H_{3}(\nu)+\frac{s_{*}^{2}}{\gamma_{\mathrm{s}}^{2}} H_{1}(\nu) k^{2}\right] W_{\mathrm{s}}(k s),
$$

\footnotetext{
${ }^{3}$ The small differences of our value of $\kappa$ and Bower et al.'s are due to different fitting formula for power spectrum.

${ }^{4}$ For interested readers, we refer Appendix E of Matsubara (1995) which contains the essential equations to derive the following equation. One can also show that the higher-order kernels $\widetilde{K}^{(n)}$ of this model are finite in large-scale limit.
} 
where $W_{\mathrm{s}}(k s)$ is a Fourier transform of smoothing function for $\delta_{\mathrm{L} s}$. Other quantities in this expression are defined by

$$
\begin{aligned}
& \sigma_{\mathrm{s} j}^{2}=\int \frac{k^{2} d k}{2 \pi^{2}} k^{2 j} P_{\mathrm{L}}(k) W_{\mathrm{s}}^{2}(k s), \\
& \nu=\frac{\delta_{\mathrm{t}}}{\sigma_{\mathrm{s} 0}}, \quad s_{*}=\sqrt{3} \frac{\sigma_{\mathrm{s} 1}}{\sigma_{\mathrm{s} 2}}, \quad \gamma_{\mathrm{s}}=\frac{\sigma_{\mathrm{s} 1}^{2}}{\sigma_{\mathrm{s} 2} \sigma_{\mathrm{s} 0}} .
\end{aligned}
$$

Hermite polynomial $H_{n}$ are defined with the normalization,

$$
H_{n}(\nu)=e^{\nu^{2} / 2}\left(-\frac{d}{d \nu}\right)^{n} e^{-\nu^{2} / 2} .
$$

Equation (3.13) explicitly show the scale-dependence of bias parameter in Fourier space. The scale of nonlocality corresponds to $s_{*}$, which is of order of smoothing length $s$ for obtaining density peaks.

The stochastic parameters $b(R), r(R)$ in linear regime for density peaks are derived from the equation (2.38). Assuming $\nu>1$, the result is

$$
\begin{aligned}
& b(R)=\frac{\nu}{\nu^{2}-1} \frac{\sigma_{\mathrm{c} 0}}{\sigma_{0} \sigma_{\mathrm{s} 0}} \sqrt{\left(\nu^{2}-3\right)^{2}+6\left(\nu^{2}-3\right) \frac{\gamma_{\mathrm{c}}^{2}}{\gamma_{\mathrm{s}}^{2}}\left(\frac{s_{*}}{c_{*}}\right)^{2}+9 \frac{\gamma_{\mathrm{c}}^{2}}{\gamma_{\mathrm{s}}^{4}}\left(\frac{s_{*}}{c_{*}}\right)^{4}}, \\
& r(R)=\frac{\sigma_{\mathrm{d} 0}^{2}}{\sigma_{0} \sigma_{\mathrm{c} 0}} \frac{\nu^{2}-3+3 \frac{\gamma_{\mathrm{d}}^{2}}{\gamma_{\mathrm{s}}^{2}}\left(\frac{s_{*}}{d_{*}}\right)^{2}}{\sqrt{\left(\nu^{2}-3\right)^{2}+6\left(\nu^{2}-3\right) \frac{\gamma_{\mathrm{c}}^{2}}{\gamma_{\mathrm{s}}^{2}}\left(\frac{s_{*}}{c_{*}}\right)^{2}+9 \frac{\gamma_{\mathrm{c}}^{2}}{\gamma_{\mathrm{s}}^{4}}\left(\frac{s_{*}}{c_{*}}\right)^{4}}},
\end{aligned}
$$

where spectral indices of various kind are defined as follows:

$$
\begin{array}{ll}
\sigma_{\mathrm{c} j}^{2}=\int \frac{k^{2} d k}{2 \pi^{2}} k^{2 j} P_{\mathrm{L}}(k) W_{\mathrm{s}}^{2}(k s) W^{2}(k R), & c_{*}=\sqrt{3} \frac{\sigma_{\mathrm{c} 1}}{\sigma_{\mathrm{c} 2}}, \quad \gamma_{\mathrm{c}}=\frac{\sigma_{\mathrm{c} 1}^{2}}{\sigma_{\mathrm{c} 2} \sigma_{\mathrm{c} 0}}, \\
\sigma_{\mathrm{d} j}^{2}=\int \frac{k^{2} d k}{2 \pi^{2}} k^{2 j} P_{\mathrm{L}}(k) W_{\mathrm{s}}(k s) W^{2}(k R), & d_{*}=\sqrt{3} \frac{\sigma_{\mathrm{d} 1}}{\sigma_{\mathrm{d} 2}}, \quad \gamma_{\mathrm{d}}=\frac{\sigma_{\mathrm{d} 1}^{2}}{\sigma_{\mathrm{d} 2} \sigma_{\mathrm{d} 0}} .
\end{array}
$$

Equations (3.17) and (3.18) describe stochastic parameters in real space.

It is known that if we take both high threshold limit, $\nu \rightarrow \infty$, and large scale limit, $R \rightarrow \infty$, the correlation function of the peak model reduces to that of linear bias, $\xi_{\mathrm{gg}} \sim\left(\nu / \sigma_{0}\right)^{2} \xi_{\mathrm{mm}}$ (Kaiser 1984; BBKS 1986). This property is easily confirmed from equation (3.17), where $\sigma_{\mathrm{c} 0}=\sigma_{\mathrm{d} 0}=\sigma_{0}$ for $R \rightarrow \infty$.

In Figure 2, these equations are plotted for $\nu=3.0$ with $s=1,5,10 h^{-1} \mathrm{Mpc}$, where we assume CDM power spectrum of BBKS, equation (3.9), with primordial spectral index 
$n=1$ and shape parameter $\Gamma=0.25$ and linear amplitude $A=1.591 \times 10^{6}\left(h^{-1} \mathrm{Mpc}\right)^{4}$ which corresponds to $\sigma_{8}=1$. The top-hat smoothing function is applied for smoothing $R$, while the Gaussian smoothing function is applied for smoothing $s$ :

$$
W(x)=\frac{3}{x^{3}}(\sin x-x \cos x), \quad W_{\mathrm{s}}(x)=e^{-x^{2} / 2} .
$$

The large smoothing lengths, $s=5,10 h^{-1} \mathrm{Mpc}$ do not correspond to galaxy formation, but rather they correspond to cluster formation, since the cluster of galaxies are density peaks of large smoothing length.

As seen in the Figure, once the smoothing scale $R$ exceeds the smoothing length $s$, which is the scale of galaxy or cluster formation in this model, the parameter $r$ of stochasticity rapidly converges to unity, which means there is no stochasticity above that scale. As stochasticity vanishes, the bias parameter $b$ converges to a constant on large scales.

\section{DISCUSSION AND CONCLUSIONS}

In this paper we explicitly derive the stochasticity parameters of the bias in linear regime from the nonlocality of the galaxy formation. By using the generalized WienerHermite functionals, we can derive the two-point correlation on linear scales which is valid even if the galaxy formation process itself is both nonlinear and nonlocal. This is in contrast to the usual Taylor expansion which can not treat the strongly nonlinear features of galaxy formation. Wiener-Hermite functionals are orthogonal functionals and we do not have to assume the smallness of $\delta_{\mathrm{L}}$ itself, and even do not have to know the exact nonlinear evolution of density contrast, $\delta_{\mathrm{m}}$. Instead, we assume only the smallness of correlation function on large scales.

We show that the stochasticity in Fourier space does not exist in linear regime (except for the constant term which comes from the small scale behavior of correlation function), and that the biasing parameter in Fourier space $b_{\mathrm{F}}(k)$ is given by $\widetilde{K}_{\mathrm{g}}^{(1)}(k)$. This conclusion is true as long as the galaxy formation process satisfies the relation, $\widetilde{K}_{\mathrm{g}}^{(1)}(k) \neq 0$, and higher-order kernels $\widetilde{K}^{(n)}(n \geq 2)$ do not increase with scales when $k \rightarrow 0$. This property in Fourier space is simply because the galaxy-galaxy and galaxy-mass correlation functions can be expressed as convolutions of mass correlation function at lowest order of the expansion by $\xi_{\mathrm{L}}$ [equation (2.29)]. A local model of galaxy formation has a constant bias factor, while the nonlocal model has a scale-dependent one, besides mode-mode coupling. In the linear regime where mode-mode coupling is negligible, stochasticity in real space comes simply from the scale-dependence of the biasing when the galaxy formation is nonlocal 
and $\widetilde{K}_{\mathrm{g}}^{(1)}(k) \neq 0$. Thus, naive introduction of stochasticity in Fourier mode in the linear regime should be avoided. One cannot introduce stochasticity in Fourier mode in the linear regime simply because of the lack of knowledge about galaxy formation. If there is any stochasticity in Fourier mode in the linear regime, it means that there are exotic process in the galaxy formation which does not come solely from the initial density field and such process should be correlated on linear scales, unless galaxy formation process has a special form to satisfy $\widetilde{K}_{\mathrm{g}}^{(1)}(k)=0$. Such kind of exotic process is not likely, at least in the present framework of the standard theory of structure formation in the universe.

We should note our analyses are restricted to the linear regime. In the nonlinear regime, there are mode coupling from both nonlinearity of density evolution and nonlinearity of galaxy formation and it makes the Fourier mode stochastic. In the region where stochasticity is prominent, the nonlinear density evolution, which is difficult to track analytically, is also prominent, so that the phenomenological approach of stochastic bias should be effectively applied in nonlinear regime (Dekel \& Lahav 1998).

In strongly nonlinear regime, phenomenological approach by the (hyper) extended perturbation theory (Colombi et al. 1997; Scoccimarro \& Frieman 1998) can shed light on how nonlinearity makes cross correlation $r$ deviate from unity. In this theory, the higher order cumulants of mass density field is given by $\left\langle\delta^{n}\right\rangle_{\mathrm{c}}=S_{n} \sigma^{2 n-2}$, where $S_{n}$ is a constant predicted by tree-level perturbation theory, and $\sigma^{2}=\left\langle\delta^{2}\right\rangle$. Although this theory contains an extrapolation of weakly nonlinear result to strongly nonlinear regime, it phenomenologically describe the numerical results. In strongly nonlinear regime, a mere averaging and cumulant are approximately equivalent in this ansatz: $\left\langle\delta^{n}\right\rangle=\left\langle\delta^{n}\right\rangle_{\mathrm{c}}+\mathcal{O}\left(\sigma^{2 n-4}\right)=S_{n} \sigma^{2 n-2}+\mathcal{O}\left(\sigma^{2 n-4}\right)$. Thus, if $\delta_{\mathrm{g}}=\delta^{n}-\left\langle\delta^{n}\right\rangle$, one can obtain $\left\langle\delta_{\mathrm{g}}^{2}\right\rangle=S_{2 n} \sigma^{4 n-2}+\mathcal{O}\left(\sigma^{4 n-4}\right)$ and $\left\langle\delta_{\mathrm{g}} \delta\right\rangle=S_{n+1} \sigma^{2 n}+\mathcal{O}\left(\sigma^{2 n-2}\right)$. Finally, one has $r=S_{n+1} / \sqrt{S_{2 n}}$. This value of $r$ depends on the scale, and departs significantly from unity on small scales.

The conclusion that the stochasticity is weak on linear scales is good news for determining the redshift distortion parameter $\beta=\Omega^{0.6} / b$ on linear scales from a redshift survey. The linear redshift distortion of power spectrum is given by, in the plane-parallel limit (Kaiser 1987; Pen 1998),

$$
P_{\mathrm{g}}^{(s)}(k)=\left[1+2 r(k) \frac{\Omega^{0.6}}{b(k)} \mu^{2}+\left(\frac{\Omega^{0.6}}{b(k)}\right)^{2} \mu^{4}\right] P_{\mathrm{g}}(k),
$$

where $P_{\mathrm{g}}^{(s)}(k)$ is redshift-space power spectrum of galaxies, and $\mu$ is a direction cosine of the angle between the wave vector $k$ and the line of sight [see Hamilton (1992) for an expression for two-point correlation function and Szalay, Matsubara \& Landy (1998) for 
its generalization to non-plane-parallel case]. Since we see $r(k)=1$ on linear scales except some special cases, we do not need to fit $r$ from the observation when we use only the linear redshift distortion. However, the previous analyses so far usually assume the bias parameter $b$ as a scale-independent constant. This assumption is justified if the scale of nonlocality of galaxy formation is actually below the linear scale. If it is not, the scale-dependence of $b(k)$ should also be determined by observation (or by theories, if possible).

The forthcoming large-scale redshift surveys will reveal the galaxy distribution especially on linear scales, on which we have not had sufficient data so far. As shown in this paper, the linear clustering properties are analytically tractable even when the galaxy formation itself is a too complex phenomenon to analytically track. The exploration of linear-scale galaxy distribution can overcome our ignorance of detailed galaxy formation processes, and will give a great insight on the primordial features of our universe.

We thank the anonymous referee for much detailed comments to improve the original manuscript. This work was supported in part by JSPS Postdoctoral Fellowships for Research Abroad. 


\section{REFERENCES}

Appel, P., \& de Fériet, J. K. 1926, Fonctions Hypergéométriques et Hypersphériques, Polynômes d'Hermite (Paris: Ganthier-Villars)

Babul, A., \& White, S. D. M. 1991, MNRAS, 253, 31P

Bardeen, J. M., Bond, J. R., Kaiser, N. \& Szalay, A. S. 1986, ApJ, 304, 15

Blanton, M., Cen, R., Ostriker, J. P., \& Strauss, M. A. 1998, ApJ, submitted, astro-ph/9807029

Bond, J. R., Cole, S, Efstathiou, G., \& Kaiser, N. 1991, ApJ, 379, 440

Bower, R. G., Coles, P., Frenk, C. S. \& White, S. D. M. 1993, ApJ, 405, 403

Catelan, P., Coles, P., Matarrese, S., \& Moscardini, L. 1994, MNRAS, 268, 966

Catelan, P., Lucchin, F., \& Matarrese, S. 1988, Phys. Rev. Lett., 61, 267

Catelan, P., Lucchin, F., Matarrese, S., \& Porciani, C. 1998, MNRAS, 297, 692

Catelan, P., Matarrese, S., \& Porciani, C. 1998, ApJ, 502, L1

Cen, R. Y., \& Ostriker, J. P. 1992, ApJ, 399, L113

Cline, J. M., Politzer, H. D., Rey, S.-J., \& Wise, M. B. 1987, Commun. Math. Phys., 112,217

Cole, S., \& Kaiser, N. 1989, MNRAS, 237, 1127

Coles, P. 1993, MNRAS, 262, 1065

Colombi, S., Bernardeau, F., Bouchet, F. R., \& Hernquist, L. 1997, MNRAS, 287, 241

Davis, M., \& Geller, M. J. 1976, ApJ, 208, 13

Davis, M., Efstathiou, G., Frenk, C. S. \& White, S. D. M. 1985, ApJ, 292, 371

Dressler, A. 1980, ApJ, 236, 351

Dekel, A., \& Lahav, O. 1998, preprint, astro-ph/9806193

Fry, J. N. 1984, ApJ, 279, 499

Fry, J. N. \& Gaztañaga, E. 1993, ApJ, 413, 447

Giovanelli, R., Haynes, M. P., \& Chincarini, G. L. 1986, ApJ, 300, 77 
Guzzo, L., Strauss, M. A., Fisher, K. B., Giovanelli, R., \& Haynes, M. P. 1997, ApJ, 489,37

Hamilton, A. J. S. 1992, ApJ, 385, L5

Hermit, S., Santiago, B. X., Lahav, O., Strauss, M. A., Davis, M., Dressler, A., \& Huchra, J. P. 1996, MNRAS, 283, 709

Jensen, L. G., \& Szalay, A. S. 1986, ApJ, 305, L5

Jing, Y.-P. 1998, ApJ, 503, L9

Kaiser, N. 1984, ApJ, 284, L9

Kaiser, N. 1987, MNRAS, 227, 1

Loveday, J., Efstathiou, G., Maddox, S. J., \& Peterson, B. A. 1996, ApJ, 468, 1

Matsubara, T. 1995, ApJS, 101, 1

Mann, R. G., Peacock, J. A., \& Heavens, A. F. 1998, MNRAS, 293, 209

Mo, H. J., \& White, S. D. M. 1996, MNRAS, 282, 347

Narayanan, V. K., Berlind, A. A., \& Weinberg D. H. 1998, ApJ, submitted, astro-ph/9812002

Otto, S., Politzer, H. D., \& Wise, M. B. 1986, Phys. Rev. Lett., 56, 1878

Peebles, P. J. E. 1980, The Large-Scale Structure of the Universe (Princeton University Press: Princeton)

Pen, U. 1998, ApJ, 504, 601

Press, W. H., \& Schechter, P. 1974, ApJ, 187, 425

Rees, M. J. \& Ostriker, J. P. 1977, MNRAS, 179, 541

Santiago, B. X., \& Strauss, M. A. 1992, ApJ, 387, 9

Scherrer, R. J. \& Weinberg, D. H. 1998, ApJ, 504, 607

Scoccimarro, R., \& Frieman, J. A. 1998, astro-ph/9811184,

Szalay, A. S. 1988, ApJ, 333, 21

Szalay, A. S., Matsubara, T. \& Landy, S. D. 1998, ApJ, 498, L1

Taruya, A., Koyama, K., \& Soda, J. 1998, ApJ, in press, astro-ph/9807005 
Taruya, A., \& Soda, J. 1998, ApJ, submitted, astro-ph/9809204

Tegmark, M., \& Blomley, B. C. 1998, ApJL, submitted, astro-ph/9809324

Tegmark, M., \& Peebles, P. J. E. 1998, ApJ, 500, L79

White, S. D. M., \& Frenk, C. S. 1991, ApJ, 379, 52 


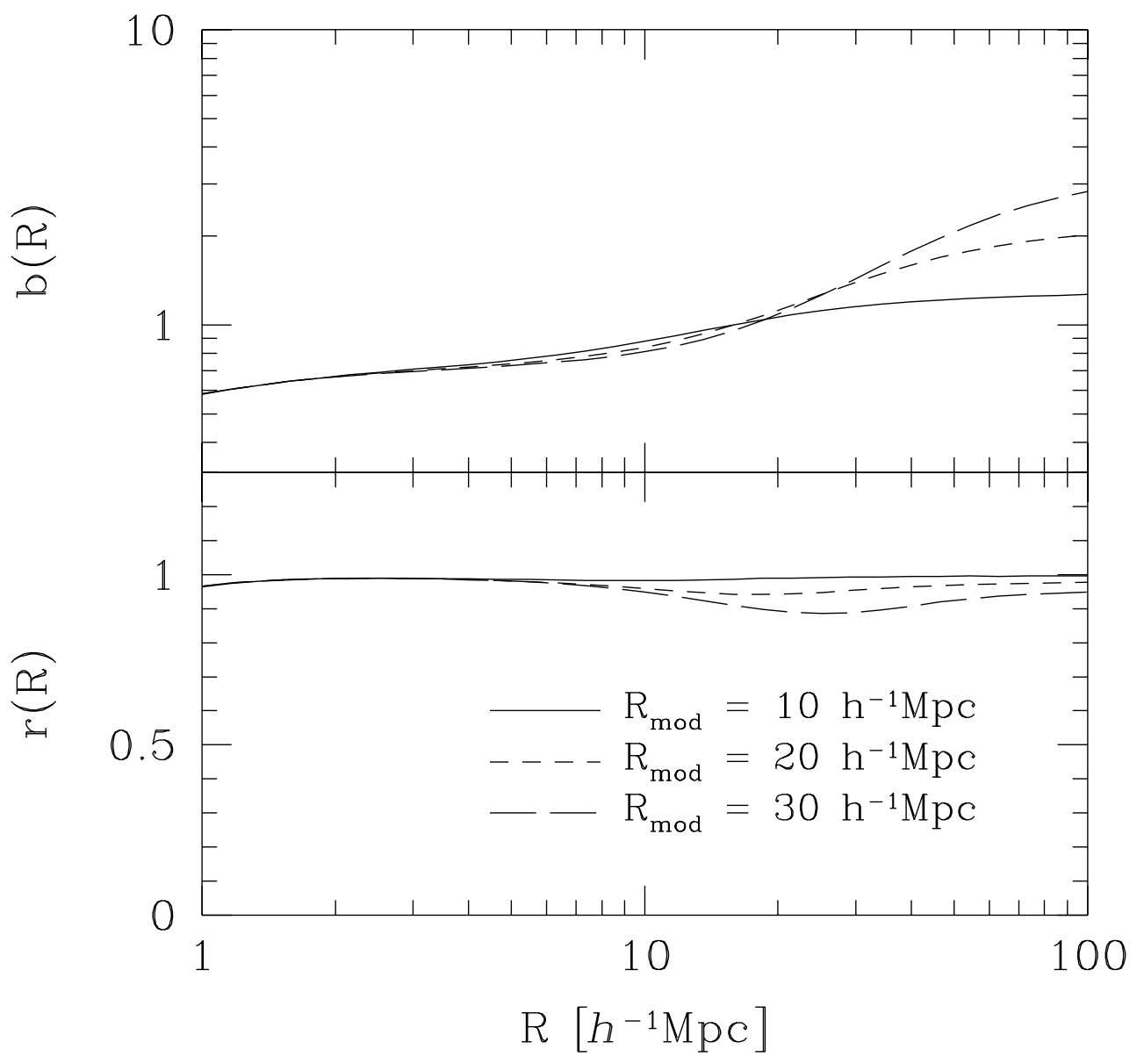

Fig. 1. - The stochastic bias parameters $b(R)$ and $r(R)$ for the cooperative model are shown. Underlying density fluctuation is assumed by CDM model with shape parameter $\Gamma=0.5$ and amplitude $\sigma_{8}=1$. Gaussian smoothing scale and the threshold are fixed as $s=0.5 h^{-1} \mathrm{Mpc}, \nu=2.8$, while the three different modulation scale $R_{\text {mod }}=10 h^{-1} \mathrm{Mpc}$ (solid line), $20 h^{-1} \mathrm{Mpc}$ (dashed line), $30 h^{-1} \mathrm{Mpc}$ (long-dashed line) are plotted. The modulation coefficient is adjusted so as to give a $2.5 \%$ rms modulation of the threshold: $\kappa=0.89,2.32$, and 4.35 , respectively. 


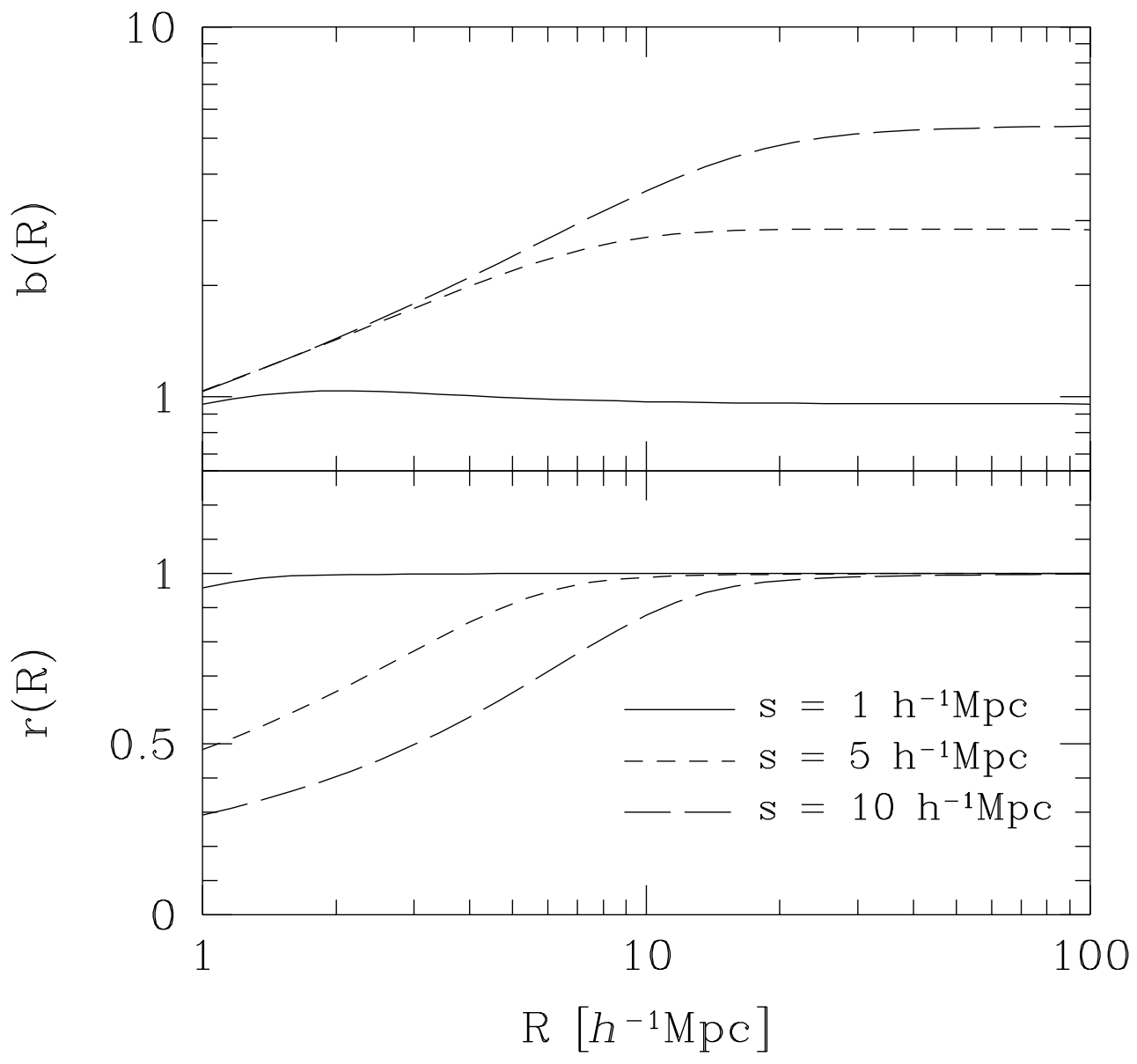

Fig. 2.- The stochastic bias parameters $b(R)$ and $r(R)$ for a peak model are shown. Gaussian smoothing scale $s$ which defines density peaks are varied as $s=1,5,10 h^{-1} \mathrm{Mpc}$, which are displayed by solid lines, short dashed lines, and long dashed lines, respectively. The threshold for density peaks is fixed as $\nu=3.0$. 